\title{
MIIASI UMANA: CASO CLINICO
}

\section{ARTICOLO ORIGINALE}

SILVA, Agnaldo Plácido da ${ }^{1}$, PLÁCIDO, Eloá Jessica Mendes dos Santos², MORAES, Walber Breno de Souza ${ }^{3}$

SILVA, Agnaldo Plácido da. PLÁCIDO, Eloá Jessica Mendes dos Santos. MORAES, Walber Breno de Souza. Miiasi umana: Caso clinico. Revista Científica Multidisciplinar Núcleo do Conhecimento. Anno 05, Ed. 08, Vol. 10, pp. 39-46. nell'agosto 2020. ISSN: 2448-0959, collegamento di accesso: https://www.nucleodoconhecimento.com.br/salute/miiase-umana, DOI: 10.32749/nucleodoconhecimento.com.br/salute/miiase-umana

\section{RIEPILOGO}

La miiasi è considerata un'infestazione di dermatozoonosi causata dall'infestazione di larve di dipteran nei tessuti o negli organi, che depongono le loro uova negli esseri umani o negli animali, che per un certo periodo si nutrono di tessuti vivi o morti dell'ospite la presenza di miiasi nella cavità orale può essere considerata una cosa rara. Questo tipo di malattia colpisce più spesso persone di basso status socioeconomico, immunocompromato, anziani costretti a letto e con disturbi psichiatrici. Grazie al suo grande potenziale distruttivo, una prevenzione e un trattamento appropriati tempestivi e importanti, c'è anche poca conoscenza del professionista dentale per la diagnosi e il trattamento di tale patologia, Per questo motivo, il presente studio riporta un caso clinico di miiasi orale in una persona anziana costretta a letto con una storia di lesione maligna laringe/glottal neoplasia, presentando debolezza fisica e mentale e inadeguata igiene del corpo e mancanza

\footnotetext{
${ }^{1}$ Dottorando in Scienze Biomediche presso IUNIR - Istituto Universitario Italiano di Rosario - Argentina. Specialista in Chirurgia e Traumatologia Bucomaxillofacciale di Ciodonto - Clinica Odontoiatrica Integrata. Laureato in odontoiatria presso ASCES - Associazione Caruaruense di Istruzione Superiore. Laureato in biologo presso UPE - Università di Pernambuco.

${ }^{2}$ Studente del Corso di Fisioterapia presso la Facoltà UNINASSAU.

${ }^{3}$ Laurea in Biologia UPE- Università di Pernambuco. Master in Risorse Naturali - UFCG. Professore presso UNOPAR.
} 
di tenuta delle labbra che ha portato all'infestazione della lingua. La diagnosi è stata clinicamente stabilita sulla base dell'osservazione del sanguinamento della lingua, del distacco del tessuto e della presenza di larve che erano tra il secondo e il terzo fase dello sviluppo. II trattamento è stato avviato come ricovero in ospedale del paziente per debridement dei tessuti necrotici e rimozione di larve e prescrizione di ivermectina. I pazienti costretti a letto con debolezza fisica e metallica costituiscono un gruppo di rischio per la miiasi orale, ed è di grande importanza guidare gli operatori sanitari e i familiari in relazione alle cure dentistiche al fine di prevenire questa patologia. Tuttavia, la mancanza di un consenso sul miglior approccio terapeutico per i casi di miiasi orale è stata presa come condotta terapeutica per la malattia la rimozione meccanica delle larve e l'istituzione dell'uso orale dell'ivermectina.

Parole chiave: Larva, miiasi umana, mosche, salute pubblica.

\section{INTRODUZIONE}

La miiasi è definita come una patologia in cui c'è pestilenza delle larve di mosche che si nutrono di tessuti viventi o morti (VINIT; JAYAVELU e SHRUTHA, 2013), anche se questa parasitosi è stata descritta per molti anni dai ricercatori, il termine tecnico per designare la bicheira è stato suggerito nel 1840 dal reverendo F. W. Hope nel suo lavoro "On insects and their larvae occasionally found in the human body", dove si propone l'uso del termine "myiasis", che deriva dalle parole greche myia (volare) e ase (malattia) (GUIMARAES; PAPAVERO e PRADO, 1983). Nella letteratura indù la miiasi era considerata dalla mitologia come punizione dei peccatori (PARMAR; DAVESSAR et al., 2018).

I pazienti con miiasi presentano generalmente alcuni fattori che portano alla predisposizione al loro sviluppo, come il ritardo mentale, disturbi psichiatrici, senilità, malattie vascolari, neoplasmi, diabete e immunodepressione e consumo cronico di alcol, e si verifica preferibilmente negli anziani. (MARQUEZ ; MATTOS e NASCIMENTO, 2007) (SILVEIRA; PINHEIRO et al., 2015) Tuttavia, i fattori di 
maggiore rilevanza per l'insorgenza della Miiasi sono ancora il verificarsi di lesioni esposte con esudazioni e scarsa igiene corporea e orale (NASCIMENTO; OLIVEIRA et al., 2005) (TOURÉ, 1994).

Si tratta di una situazione di gravità fatale per il paziente, oltre a causare dolore e distruzione dei tessuti. La miiasi orale può presentarsi come lacerazione della cavità orale, gonfiore della mucosa orale, infiammazione del gingival (RIBEIRO; PEREIRA et al., 2001). "Nel caso della cavitaria di Miiasis, che comprende le regioni della bocca e del naso e del seno paranasale, si può affermare che si tratta di un tipo molto raro di miiasi" (BARNA- FERRA e altni, 2016). La varietà più diffusa di specie di mosca responsabili della maggior parte dei casi di miiasi include Cochliomyia hominivorax, Chrysomya bezziana, Oestrus ovis, Magnificent Wohlfahrtia e la Musca domestica. L'incidenza dell'infezione è rara anche nei paesi in via di sviluppo (KHAN; SABIR et al., 2018) (TOURÉ, 1994).

II trattamento per la miasi consiste essenzialmente nell'asportazione manuale delle larve, con l'ausilio di curette e pinzette cliniche, generalmente in anestesia o analgesia, a seconda delle condizioni cliniche del paziente, l'asportazione delle larve deve essere eseguita con cura, in modo che il le larve non vengono frammentate, e i loro resti rimangono al loro posto, generando un processo infettivo (CAVALCANTI, 2008), anche se esistono sul mercato alcuni prodotti che possono essere utilizzati per facilitarne la rimozione (BARNABÉ; FERRAZ et al., 2016), la letteratura riporta con grande successo l'uso sistemico di una dose di ivermectina, antibiotico macrolide semisintetico per il trattamento dei casi più gravi che comportano l'esistenza di miasi orale, dimostrandosi un metodo sicuro ai dosaggi raccomandati di $200 \mu \mathrm{g} / \mathrm{kg}$. Anche a dosaggi di $300 \circ 400 \mu \mathrm{g} / \mathrm{kg}$, i pazienti in trattamento non presentavano alcun effetto collaterale clinico importante, ad eccezione di un lieve prurito (GEALH; FERREIRA et al., 2009) (RIBEIRO; PEREIRA et al., 2001).

Possiamo classificare la Miiasi in tre tipi diversi: biontofago o primaria che sono quelle larve dipteran che si sviluppano su o allinterno dei vertebrati, nutrendosi dei tessuti dell'ospite vivente. Tra queste specie, possiamo trovare Cochliomy homini 
vorax e Dermatobia ominis. Necrobiollins secondari sono quelli in cui le larve dipteran si sviluppano in materia organica che è già decomposizione (cadaveri), agendo come saprofago, ma può anche influenzare i tessuti necrotici in un ospite vivente. I generi più comuni sono: Lucalia, Faenicia, Musca e Fannia. Pseudomiiasi (accidentale): ciò può verificarsi per ingestione di ovuli e/o larve dipteran presenti negli alimenti contaminati ingeriti dall'ospite, causando un disturbo intestinale (CAVALCANTI, 2008) (BOROS; BOROS et al., 2006).

\section{CASO CLINICO}

Un paziente maschio di 69 anni, diagnosticato con neoplasma maligno nella lingua (sottoposto a chemioterapia) è stato ricoverato al pronto soccorso dell'Ospedale Regionale Dom Moura di Garanhuns - Pernambuco lamentando dolore e infrequenza nella regione della bocca per diverse settimane, è stato sottoposto al servizio di Chirurgia e Traumatologia Bucomaxillofacciale. Al momento del ricovero, il paziente era afebrile, orientato e cooperativo, sanguinamento è stato osservato in bocca, considerando il rapporto della famiglia che il paziente aveva problemi dentali, e gli esami sono stati eseguiti. Nella valutazione intraorale ha mostrato una lesione ulcerosa ben definita con tessuto necrotico centrale e diverse larve vive sulla lingua. (Figura 1) 
Figura 1 - Larve sulla lingua.

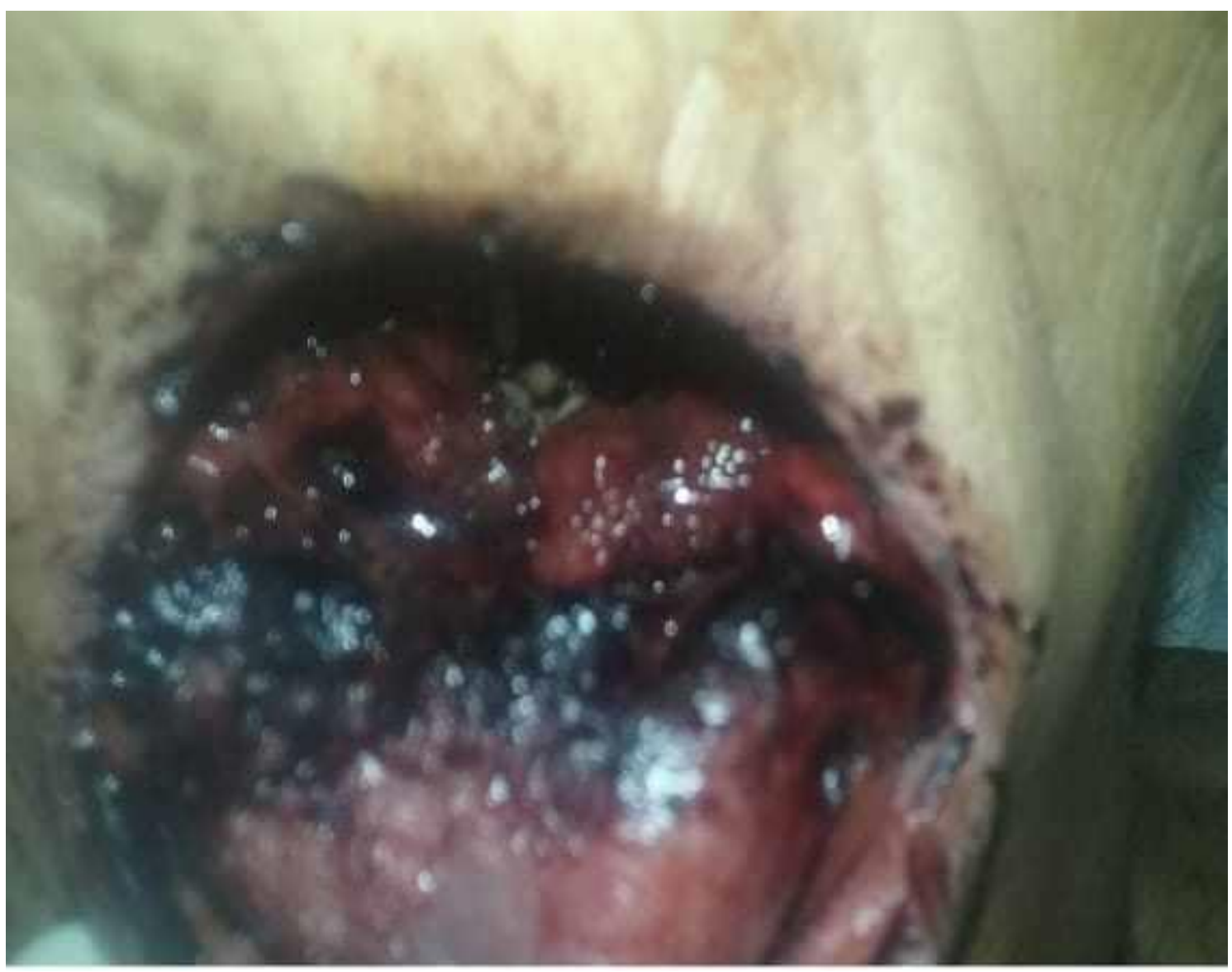

Fonte: Gli autori (2020).

II paziente affetto dalla malattia presentava fattori predisponezionali come debolezza fisica e mentale, disidratazione, igiene del corpo inadeguata, diabete, malnutrizione, respirazione della bocca durante il sonno e scarsa manutenzione dell'igiene orale.

Così, è stato eseguito il trattamento sintomatico primario. La ferita è stata pulita e disinfettata con soluzione di Chlorhexidine dello 0,12\%. Le larve sono state rimosse manualmente in anestesia locale con l'aiuto di pinzette cliniche, l'intero campo asetico, le larve in questo caso sono state raccolte e collocate in un vassoio clinico. Circa 80 larve sono state rimosse dalla regione e non è stato possibile rimuovere tutte (Figura 2). 
Figura 2 - Larve rimosse dalla lesione

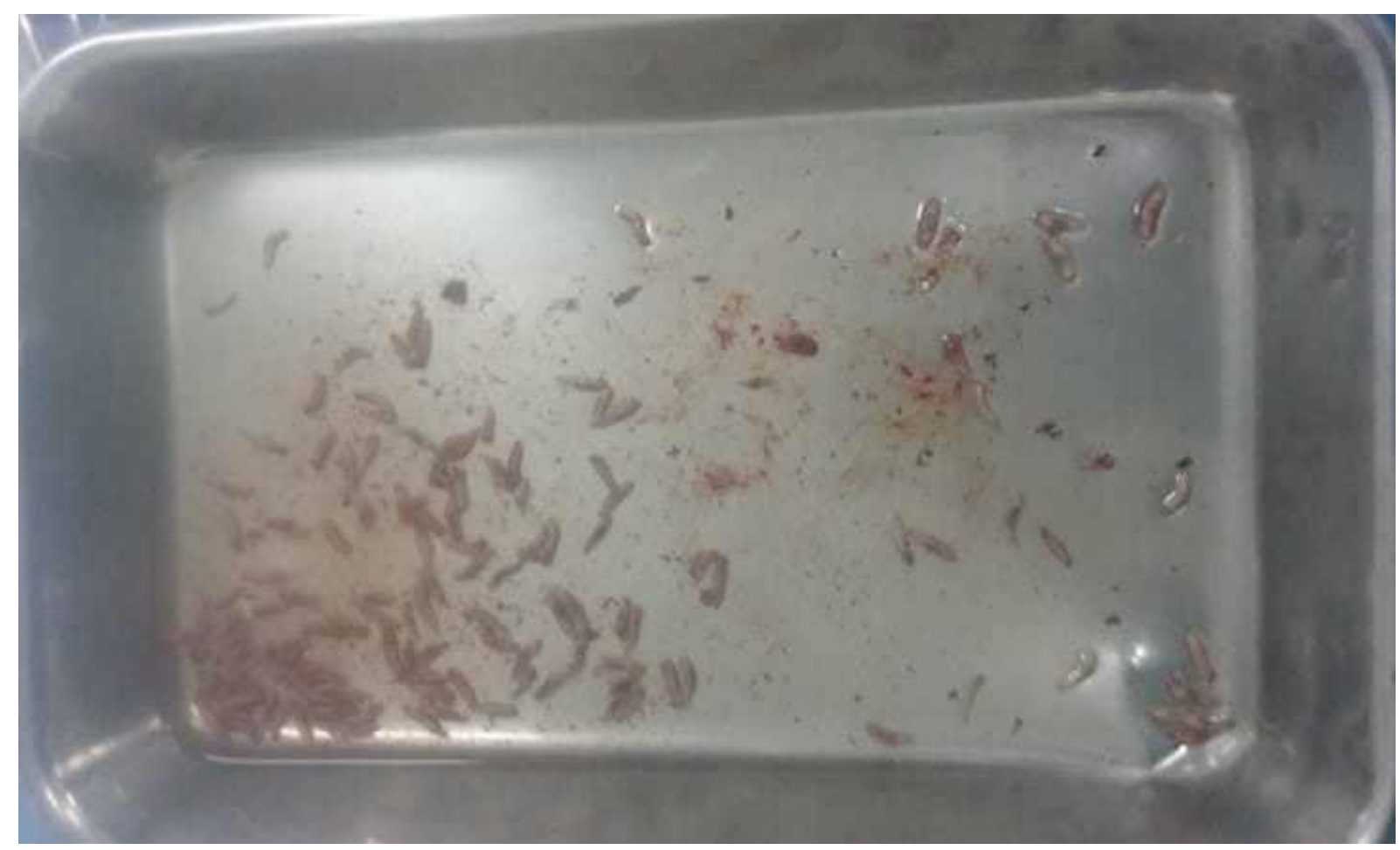

Fonte: Gli autori (2020).

Il paziente è stato ricoverato in ospedale e la terapia antibiotica per via endovenosa è stata avviata Cephalotina 1g. Dipirona e Ketoprofene, e una singola dose orale di Ivermectin due compresse, cioè, $12 \mathrm{mg}$ (circa $200 \mathrm{~g} / \mathrm{kg}$ ), Dopo 10 giorni tutte le larve rimanenti erano state sradicate, senza bisogno di complemento di Ivermectin.

\section{DISCUSSIONE}

Durante il processo di sviluppo delle larve, i tessuti adiacenti presentano un processo infiammatorio, con o senza ulcerazione o necrosi dei tessuti (CARVALHO; SANTOS et al., 2008). Molte gravi complicazioni possono verificarsi a causa della miiasi: cellulite, erosione delle ossa e dei denti, batteriemia e morte (SAKACS e MACPHERSON, 2007).

In molti degli articoli consultati, abbiamo osservato che gli autori menzionano che la miiasi orale è una patologia rara e che i dipterani non hanno alcuna preferenza per 
l'età, il sesso e l'etnia. E che l'infestazione della larva dei dipterani è più nella zona rurale, infestando animali domestici come cani, gatti, maiali, buoi. L'opinione generale degli autori è che le persone che sono state infestate dalle larve sono mentalmente handicappati, le persone con poca igiene corporea e orale, come il basso livello di istruzione, le persone a basso status socioeconomico (BOROS; BOROS et al., 2006).

La diagnosi di miiasi umana è clinica, in modo che si possono rilevare alcune delle sue complicazioni possono essere necessarie per eseguire la tomografia computerizzata, ultrasuoni o risonanza magnetica (SHENOUDA; ENTEN et al., 2018).

La presenza di larve sulla pelle innesca una risposta infiammatoria locale con la migrazione e la proliferazione di cellule infiammatorie come neutrofili, mastocite, eosinofili, fibroblasti e cellule endoteliali. Tuttavia, il numero completo di cellule del sangue può mostrare alti livelli di leucociti ed eosinofili (PAYNE e COSGROVE, 1966) (SHENOUDA; ENTEN et al., 2018).

Alcuni autori sostengono un trattamento convenzionale della miiasi, che include la rimozione delle larve con l'aiuto di sostanze chimiche, che mira a promuovere l'asfissia delle larve inducendo la loro uscita dalla lesione. Nelle lesioni più grandi in cui presenta un quadro clinico di cellulite adiacente, un debridement dei tessuti locali sarebbe indicato con la rimozione dei tessuti necrotici, ma altri difendono come ideale, il trattamento chirurgico, che consente la rimozione di tutte le larve alleate a un debridement, e antibiotici per prevenire l'infezione secondaria, un altro metodo sono già stati provati come l'uso di una singola dose di ivermectina (PARMAR; DAVESSAR et al., 2018).

La miiasi può essere vista come un problema di salute pubblica che può essere prevenuto eliminando i fattori di rischio per la salute umana, come la mancanza di servizi igienico-sanitari di base e lo smaltimento inadeguato dei rifiuti, dove viene esposto materiale organico, che attira insetti e piccoli animali, creando un ciclo 
sostenibile di sporcizia. La scarsa igiene è probabilmente il fattore di rischio più importante per la miiasi umana (FRANCESCONI e LUPI, 2012).

\section{CONCLUSIONE}

Nella letteratura sono stati proposti diversi trattamenti per la miiasi cavitaria, che vanno dall'estrazione meccanica all'uso di sostanze topice, orali ed endovenose. Ma una diagnosi precoce e corretta permette un trattamento con una prognosi favorevole. Tuttavia, qualunque siano i mezzi impiegati, è essenziale che il paziente sia orientato all'adozione di adeguate misure igieniche personali. Tuttavia, non vi è consenso sul miglior approccio terapeutico per i casi di miiasi orale.

\section{RIFERIMENTI}

BARNABÉ, A. S. et al. Epidemiologia Da Miíase Cutânea: Revisão Da Literatura. Atas de Ciências da Saúde, São Paulo, v. 4, n. 2, p. 14-22, Abril - Junho 2016.

BOROS, L. F. et al. Míase Labial. Revista Dens, v. 14, n. 1, p. 1-10, Revista Dens, v.14, n.1, maio/outubro 20062006.

CARVALHO, R. W. F. et al. Oral and maxillofacial myiasis associated with epidermoid carcinoma: a case report. Journal of Oral Science, v. 50, n. 1, p. 103-105, 2008.

CAVAlCANTI, A. L. Mí́ase Oral: etiologia, diagnóstico e tratamento. Rev. Fac. Odontol. Porto Alegre, Porto Alegre, v. 49, n. 2, p. 32-35, maio/ago. 2008.

CAVAlCANTI, A. L. Mí́ase Oral: etiologia, diagnóstico e tratamento. Rev. Fac. Odontol. Porto Alegre, Porto Alegre, v. 46, n. 2, p. 32-35, maio/ago. 2008.

FRANCESCONI, ; LUPI,. Myiasis. Clinical Microbiology Reviews , v. 25, n. 1, p. $79-$ 105, Jan. 2012. 
GEALH, W. C. et al. Treatment of oral myiasis caused by Cochliomyia hominivorax: two cases treated with ivermectin. British journal of Oral and Maxillofacial Surgeons, v. 47 , n. 1, p. 23-26, January 2009.

GUIMARAES, J. H.; PAPAVERO, ; PRADO, A. P. D. As Mi1ases Na Região Neotropical. Revista Brasileira De Zoologia (Identificação, Biologia, Bibliografia), São Paulo, v. 1, n. 4, p. 239.416, 1983.

KHAN, B. A. et al. Oral and cutaneous myiasis in a 5 year old child from Karachi, Pakistan. Le Infezioni in Medicina, v. 4, p. 385-388, 2018.

MARQUEZ, A. T.; MATTOS, M. D. S.; NASCIMENTO, S. B. Miíases associadas com alguns fatores sócio-econômicos em cinco áreas urbanas do Estado do Rio de Janeiro. Revista da Sociedade Brasileira de Medicina Tropical, v. 40, n. 2, p. 175180, março - abril 2007.

NASCIMENTO, E. M. F. D. et al. Miíases Humanas Por Cochliomyia Hominivorax (Coquerel, 1858) (Diptera, Calliphoridae) Em Hospitais Públicos Na Cidade Do Recife, Pernambuco, Brasil. Entomología y Vectores, v. 12, n. 1, p. 37-51, 2005.

PARMAR, S. et al. Tracheostomal Myiasis: A Case Report And Review Of Literature. International Journal of Scientific Research, v. 7, n. 12, p. 4-6, December 2018.

PAYNE, J. A.; COSGROVE, G. E. Tissue changes following Cuterebra infestation in rodents. The American Midland Naturalist, v. 75, n. 1, p. 205-213, Jan. 1966.

RIBEIRO, F. A. Q. et al. Tratamento da miíase humana cavitária com ivermectina oral. Rev Bras Otorrinolaringol, v. 67, n. 6, p. 755-761, nov./dez. 2001.

SHENOUDA, M. et al. Human Botfly: A Case Report and Overview of Differential Diagnosis. J Investig Med High Impact Case Rep., v. 6, Jan-Dec; 2018.

SILVEIRA, M. A. A. et al. Cavitary myiasis mimicking peritonsilar abscess. Brazilian Journal of Otorhinolaryngology, v. 81, p. 336-338, 2015. 
SZAKACS, T. A.; MACPHERSON,. Nosocomial myiasis in a Canadian intensive care unit. CMAJ, v. 177, n. 7, p. 719-720, 25 September 2007.

TOURÉ, S. M. Les myiases d'importance économique. Rev. sci. tech. Off. int. Epiz., 1994, 13 (4), 1053-1073, v. 13, n. 4, p. 1053-1073, 1994.

VINIT, G. B. ; JAYAVELU, P.; SHRUTHA, S. P. Oral myiasis in a maxillofacial trauma patient. J Pharm Bioallied Sci., v. 5, n. 6, p. 195-197, 5 Jul 2013.

Inviato: Febbraio 2020.

Approvato: Agosto 2020. 